\title{
Giant Aortic Syphilitic Aneurysm of The Ascending Aorta with Erosion in the Chest Wall: Case Report
}

\author{
Ovidiu Stiru, Roxana Carmen Geana, Liana Valeanu, Diana Sorostinean, Mihai Goicea, Ion Iovu, \\ Mihai Stefan, Diana Tudor, Vlad Anton Iliescu \\ Department of Cardiovascular Surgery, Emergency Institute for Cardiovascular Diseases “Prof. Dr. C. C. Iliescu” Bucharest, Romania
}

\section{ABSTRACT}

Since the discovery of penicillin, the incidence of tertiary syphilis dramatically has decreased. However, cases of cardiovascular complications of syphilis still are present. Ascending aortic aneurysms are some of the most devastating complications. Nonetheless, syphilitic aortitis (SA) can appear and should be suspected in patients with syphilis and aortic aneurysm. We report a case of a 57-year-old patient with a large ascending aortic aneurysm with cartilage and rib erosion. The purpose of this article is to discuss the particular surgical aspects of this unusual case.

\section{INTRODUCTION}

Syphilis is an infectious disease with a wide variety of clinical presentations, depending on the stage of the disease. The introduction of antibiotic therapy reduced the prevalence of cardiovascular syphilis, especially in developed countries. Cardiovascular complications of syphilis are a late form of the disease, and they usually manifest in the fourth or fifth decade of life and occur after a latent period of usually 10 years to 25 years [Tavora 2006]. In the case of untreated primary syphilitic infection, the most significant cardiovascular complication is SA. This leads to aortic aneurysm formation in most cases, aortic regurgitation, or coronary ostial stenosis [Duncan 1983; Goldstein 2003].

SA is an inflammatory response to the invasion of the aortic wall by the Treponema pallidum, and it is characterized by inflammatory cell infiltration of the adventitia, obliterative endarteritis of the vasa vasorum, medial necrosis, and degeneration. In time, this results in weakening of the aortic wall, the ascending aorta being the most commonly affected segment $(50 \%)$, followed by the arch (35\%), and the descending aorta (15\%) [Heggtveit 1964]. Patients may be asymptomatic for a longer period, but when aortic aneurysms grow to higher dimensions the most common symptoms are pain and dyspnea. Large syphilitic aortic aneurysms (SAA) can

Received May 28, 2019; received in revised form fuly 1, 2019; accepted fuly 16, 2019.

Correspondence: Ovidiu Stiru, Emergency Institute for Cardiovascular Diseases "Prof. Dr. C. C. Iliescu" Sos. Fundeni 258, sector 2, 022328 Bucharest, Romania; +400722207286; fax:+400722207286 (e-mail: ovidiu_stiru@yahoo.com). cause compressions of the adjacent organs, such as the pulmonary artery, trachea, esophagus, recurrent laryngeal nerve, and may even lead to erosion or perforation of this organ [Tomey 2011; Watanabe 1967]. In some cases, SAA enlarges asymptomatically until a catastrophic fatal rupture occurs with sudden death. Computed tomographic angiography scan (CT-scan) and transthoracic echocardiography (TTE) are the most commonly used diagnostic techniques for SAA. Without surgical treatment, the mortality rate can reach $80 \%$ per year, due to the high rate of rupture of these aneurysms [Revest 2006]. The patients can be treated with antibiotics (penicillin in most cases) for 1-3 weeks by weekly injection and 1-20 days for daily use in surgical patients before operation [Yuan 2018]. Postoperatively, they can be treated with penicillin $\mathrm{G}$ for 6 months or doxycycline $100 \mathrm{mg}$ taken orally for 30 days [Yuan 2018].

\section{CASE REPORT}

A 57-year-old male was referred to our cardiac surgery clinic from a regional cardiology clinic. He was experiencing chest pain in the upper part of his right hemi-thorax for about 18 months before his hospitalization and described the pain as worsening in the last 3 months. He was referred to our clinic for further investigation and surgical treatment. He had a history of neglected arterial hypertension, dyslipidemia, and a toxic environment in his line of work. He denied smoking and admitted to moderate alcohol consumption. $\mathrm{He}$ had no family history of vascular disease or aortic bicuspid valve. Although he appeared oriented during the physical assessment, he exhibited accentuated personality traits such as astheno-neurotic tendencies. Further examination showed a good overall state with blood pressure of $145 / 80 \mathrm{mmHg}$, heart rate of $55 \mathrm{bpm}$, respiratory rate of 16 per minute, and oxygen saturation of $98 \%$. The rest of the clinical examination had normal findings.

The ECG showed signs of sinus rhythm and left ventricular hypertrophy. Chest radiography (X-ray) showed a considerably enlarged aortic silhouette overlapping almost two-thirds of the upper right lung field; no pleural effusions were seen. TTE showed a giant aneurysm of the ascending aorta, mild-tomoderate aortic regurgitation and mild tricuspid regurgitation. Furthermore, a CT-scan revealed an $18.82 \mathrm{~cm}$ diameter, 10.12 $\mathrm{cm}$ long aortic aneurysm with partial thrombosis of its lumen, exerting serious compression and erosion of the second right 

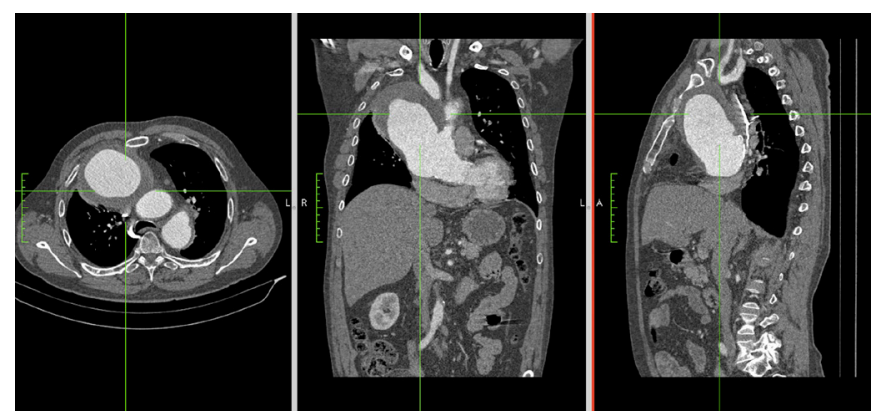

Figure 1a. Preoperative multiplanar reconstruction of angio-CT showing aneurysm of the ascending aorta.

ribs which appeared fractured in its proximal third (Figure 1a, Figure 1b). Laboratory tests on admission showed an inflammatory syndrome (CRP $24.17 \mathrm{mg} / \mathrm{L}$, Fibrinogen $479 \mathrm{mg} / \mathrm{dL}$, WBC 6040/ $\mu \mathrm{L}$ ), dyslipidemia (Triglycerides $41 \mathrm{mg} / \mathrm{dL}$, HDL Chol $32 \mathrm{mg} / \mathrm{dL}$, Total Lipid $441.5 \mathrm{mg} / \mathrm{dL}$ ), and a mild anemia (Hgb $10.6 \mathrm{mg} / \mathrm{dL}$, RBC 3.8*106/ $\mu \mathrm{L}$, HCT 32.6\%). Blood tests revealed positive results of Treponema pallidum particle agglutination (TPHA) and fluorescent treponema antibody absorption test (FTA-Abs), while the HIV test was negative. Coronarography revealed a unique nonstenotic lesion accounting for a $40 \%$ stenosis of the right coronary artery. Doppler ultrasound assessment of femoral vessels showed bilateral permeable arteries and veins, without any lesions. Taking into account the dimensions of the aneurysm and partial thrombosis of its lumen, a surgical indication is retained.

The considerable risks of the surgery regarding perioperative mortality and its possible complications, as well as the expected natural history without surgery were communicated to the patient. The patient agreed to surgical treatment. The partial thrombosis of the aneurysm enabled us to treat the patient with antibiotics (penicillin) for 3 weeks before surgery. The surgical strategy was discussed considering the CT-scan, transesophageal echocardiographic (TEE) and TTE imagery, the considerable risks regarding perioperative mortality and possible complications, and the expected natural history should surgery not proceed. Based on the symptoms, blood results and the CT-scan images, diagnosis was confirmed as SAA, and the patient was referred for elective surgery. The surgery took place in the cardiac OR, under CPB. Standard monitoring of the patient included rectal and esophageal temperature monitoring, double intra-arterial blood pressure monitoring the left radial artery and left femoral artery to have an accurate evaluation of the perfusion in the entire body and near-infrared spectroscopy (NIRS) for reflecting cerebral oxygenation of the frontal cortex to detect any potential cerebral malperfusion.

During the surgical procedure, we adopted the peripheral cardiopulmonary bypass (CPB). The common right femoral artery and right vein were cannulated (femoral cannula kit, Medtronic-DLP, Minneapolis, MN, USA), and CPB was installed with moderate hypothermia $\left(26^{\circ} \mathrm{C}\right)$. When $\mathrm{CPB}$ was established through a peripheral approach, we achieved left ventricular (LV) decompression shortly after the beginning of cooling the LV by direct cannulate through the apex

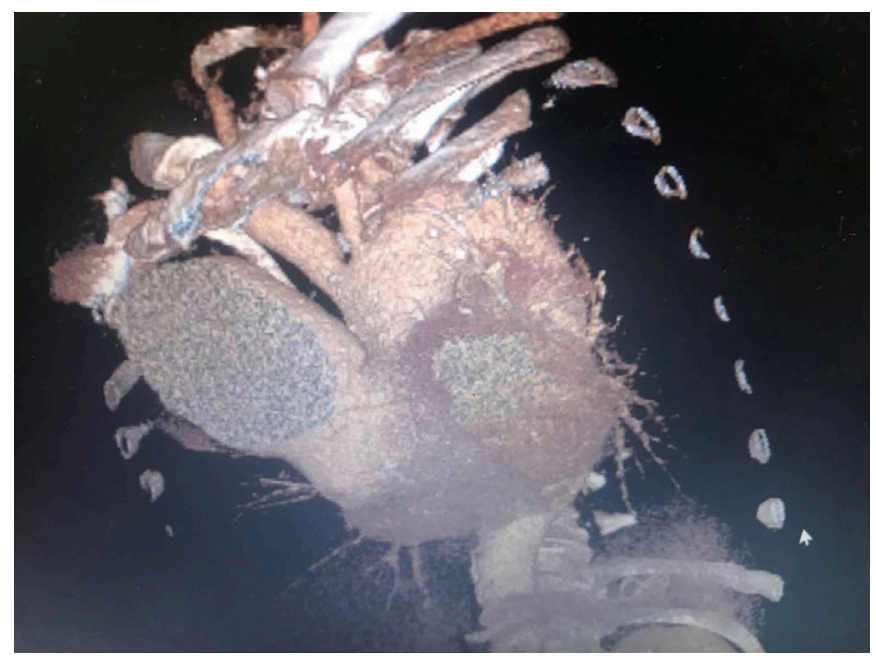

Figure 1b. Preoperative 3D reconstruction of contrast-enhanced angioCT demonstrating extensive giant sternum-eroding aneurysm of distal ascending aorta.

using a left minithoracotomy. TEE was used to precisely locate the LV apex on chest surface and cannulation was performed through the LV apex using a $26 \mathrm{Fr}$ cannula (DLP, Medtronic Inc. Minneapolis, MN). Hemostasis was obtained by pledgeted purse-string sutures. After the initiation of $\mathrm{CPB}$, the gradual cooling of the patient was initiated. When the rectal temperature reached $26^{\circ} \mathrm{C}, \mathrm{CPB}$ was interrupted to perform a median sternotomy. Due to the direct attachment of the aneurysm to the posterior side of the sternum when the sternotomy was performed, the anterior wall of the aneurysm was transected and the cavity of the aneurysm was directly exposed (Figure 2). On circulatory arrest, the aneurysm was aspirated and all clots removed from the cavity. The brain was protected using bilateral selective antegrade cerebral perfusion with a flow of $10 \mathrm{~mL} / \mathrm{kg} / \mathrm{min}$ adjusted to maintain a pump delivery pressure of 40 to $70 \mathrm{~mm} \mathrm{Hg}$, with continuous monitoring of the bilateral NIRS in the frontal cortex. In the meantime, myocardial protection was achieved with a single dose of Custodiol cardioplegia in an antegrade route directly infused into coronary ostia. The aortic valve leaflets along with the rest of the aortic root carefully were inspected, and the aortic valve was considered to be conservable. The first anastomosis performed was the distal anastomosis between the no. 32 woven Dacron graft and the distal ascending aorta. After the distal anastomosis was completed and cardiac deairing performed, the graft was clamped and CBP restarted with rewarming. Next, the proximal anastomosis was performed between the remaining end of the prosthetic graft and the sinotubular aortic junction (Figure 3).

The total time of CPB was $220 \mathrm{~min}$, the time of circulatory arrest was $61 \mathrm{~min}$, and the time of aortic clamping was $92 \mathrm{~min}$. The patient had an uneventful postoperative course, and he postoperatively was assessed by a clinical psychologist, due to difficulties in communicating with the medical staff. His neuropsychological state was evaluated as stationary compared with the period before the operation. He was 


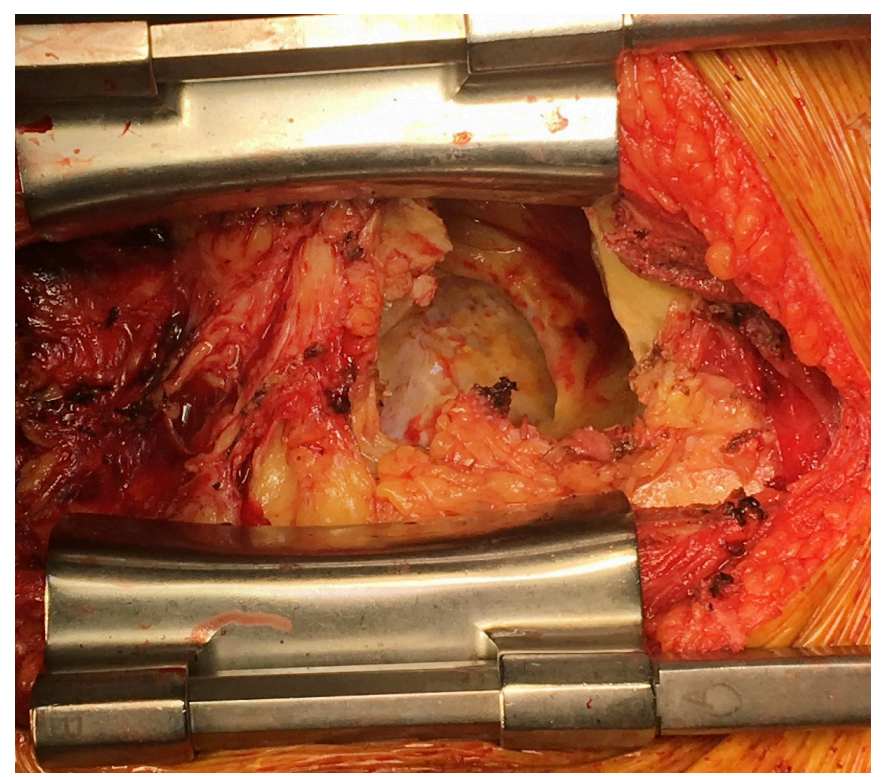

Figure 2. Intraoperative view of the direct attachment of the aneurysm to the posterior of the sternum (anterior wall of the aneurysm was transected and the cavity of the aneurysm was directly exposed).

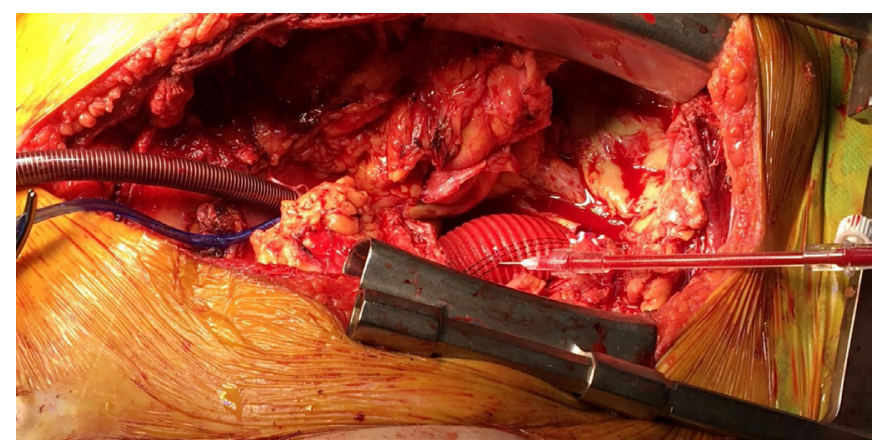

Figure 3. Intraoperative view of complete replacement of ascending aortic aneurysm with no. 32 woven Dacron graft.

discharged on the 18th day after the operation with no complications with $100 \mathrm{mg}$ doxycycline once a day for the next 30 days. A 6-month follow-up 3D CT-scan reconstruction showed no recurrence (Figure 4).

\section{DISCUSSION}

Cardiovascular complications of syphilis are a late form of the disease, and they usually manifest in the fourth to fifth decade of life, occurring after a latent period of 10 years to 25 years [Tavora 2006]. Epidemiological data show that SA may occur in $70 \%$ to $80 \%$ of untreated patients [Jakman 1989]. In the case of untreated primary syphilitic infection, the most significant cardiovascular complication is SA, which leads in most cases to aortic aneurysm formation, syphilitic aortic valvulitis with aortic regurgitation, and syphilitic coronary ostial stenosis [Duncan 1983, Goldstein 2003].

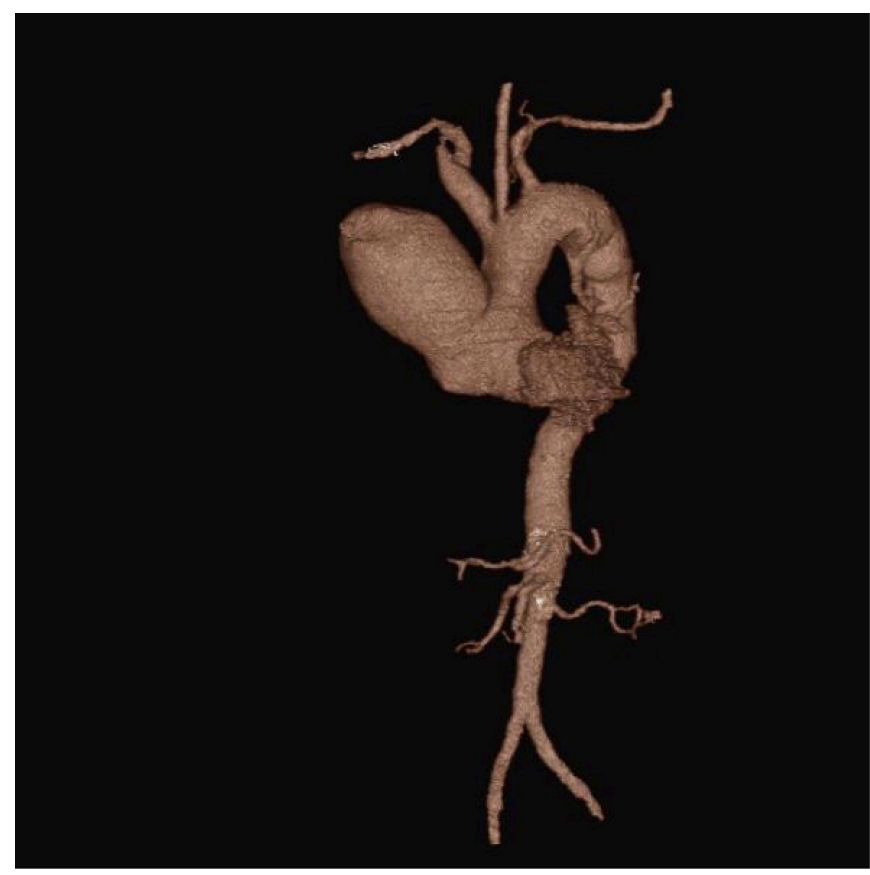

Figure 4. Postoperative 6-month reconstruction of contrast-enhanced angio-CT 3D.

Epidemiological data show that SA may occur in patients with untreated syphilis. Patients with SA may be asymptomatic for many years unless the aneurysm compresses important structures, causing acute clinical manifestations. The most common complications of SA are a rupture of the aneurysmatic ascending aorta and aortic valve insufficiency. Frequently in patients with SA apart from the ascending aorta insufficiency, the dilatation of the aortic root also can occur, leading to aortic valve insufficiency, which necessitates surgical valve replacement and aortic root reconstruction. In our case, we found a tricuspid aortic valve, mild insufficiencies without dilatation of the aortic sinotubular junction. The risk of rupture of a large aneurysm should be taken into consideration as an indication for emergency surgery. The diagnosis is confirmed using CT-scan, TTE, and serological confirmation of syphilis infection. Treponema pallidum has never been histologically demonstrated in the aortic resected wall and examination revealed predominantly lymphoplasmacytic inflammatory cell infiltrates around the vasa vasorum of the adventitia. Also, the spirochete Treponema pallidum cannot be cultured from tissue samples. When the diagnosis of syphilis aortic aneurysm is established, blood pressure should be controlled, and penicillin and anti-platelet aggregation should be given before surgical intervention.

Thoracic aortic aneurysms grow upwards at a rate of $0.10 \mathrm{~cm}$ per year [Coady 1999]. Consensus guidelines advise surgical evaluation regardless of size, for all patients with symptoms suggestive of aneurysm expansion, as well as asymptomatic individuals with an ascending aortic or aortic sinus diameter of $5.5 \mathrm{~cm}$ or greater [Hiratzka 2010]. Aneurysms are complicated because of the invasion and erosion of the chest wall, which in our case represented an 
additional surgical challenge. In such cases, unintentional rupture during the sternotomy is a cause of a surgical catastrophic complication. Mortality reported in the literature for ascending aneurysm ranges from $29 \%$ to $46 \%$ [Sullivan 1988, Revest 2006], and in most of them it is a consequence of a fatal hemorrhage due to rupture of the aneurysm during surgical repair. The complexity of this case lies in avoiding bleeding during sternotomy and maintaining proper cerebral perfusion during the circulatory arrest. In our patient, the aneurysm was adherent to the sternum, and rupture was most likely to occur during the opening of the chest. To avoid this, sternotomy was recommended to be performed when hypothermic circulatory arrest was established. During the surgical procedure, due to the huge risk of sudden rupture of the ascending aortic aneurysm at the opening of the sternum, it was recommended to adopt peripheral cardiopulmonary bypass (CPB).

One of the main limitations of peripheral CPB in the case of even mild aortic regurgitation is related to the fact that blood drainage and adequate decompression of the left ventricle (LV) are not achieved, and there can be an excessive increase of the LV pressure, which in the case of ventricular fibrillation determines subendocardial ischemia. However, when cardiopulmonary bypass is established through a peripheral approach, we achieved LV decompression shortly after the beginning of cooling the LV by direct cannulate through the apex using a left minithoracotomy. The presented case seems to offer the best solution in high-risk patients with SAA. It may be the best evidence that this is an available treatment option regarding the management of this life-threatening disease, even if it is associated with erosion of the chest wall.

\section{CONCLUSION}

SAA complicated with erosion of the chest wall is extremely rare. A CT-scan can indicate the presence of aortic aneurysm and to determine the location of the chest wall erosion. Blood tests are an effective method to clarify the etiology of the aneurysm. Surgical treatment with replacement of the entire aortic aneurysm in conjunction with CPB, circulatory arrest, moderate hypothermia, left ventricular apex venting during $\mathrm{CPB}$ cooling before sternotomy and cerebral antegrade protection, can all guarantee the success of this unusual complication.

\section{REFERENCES}

Coady MA, Rizzo JA, Hammond GL, Kopf GS, Elefteriades JA. 1999. Surgical intervention criteria for thoracic aortic aneurysms: a study of growth rates and complications. Ann Thorac Surg 67:1922-1926.

Duncan JM, Cooley DA. 1983. Surgical considerations in aortitis. Part III: syphilitic and other forms of aortitis. Tex Heart Inst J 10:337-41.

Goldstein B, Carroccio A, Ellozy SH, Spielvogel D, Morrissey N, Teodorescu V, Hollier LH, Marin ML. 2003. Combined open and endovascular repair of a syphilitic aortic aneurysm. J Vasc Surg 38(6):1422-1425.

Heggtveit HA. 1964. Syphilitic aortitis: a clinicopathologic autopsy study of 100 cases, 1950 to 1960. Circulation 24:346-55.

Hiratzka LF, Bakris GL, Beckman JA, et al. 2010. ACCF/AHA/ AATS/ ACR/ASA/SCA/SCAI/SIR/STS/SVM guidelines for the diagnosis and management of patients with thoracic aortic disease: a report of the American College of Cardiology Foundation/American Heart Association Task Force on Practice Guidelines, American Association for Thoracic Surgery, American College of Radiology, American Stroke Association, Society of Cardiovascular Anesthesiologists, Society for Cardiovascular Angiography and Interventions, Society of Interventional Radiology, Society of Thoracic Surgeons, and Society for Vascular Medicine. Circulation 121:e266-e369.

Jackman JD, RadolfJD. 1989. Cardiovascular syphilis. Am J Med 87:425-33.

Revest M, Decaux O, Frouget T, Cazalets C, Cador B, Jégo P et al. 2006. Syphilitic aortitis. Experience of an internal medicine unit. Rev Med Intern 27:16-20.

Sullivan KL, Steiner RM, Smullens SN, Griska L, Meister SG. 1988. Pseudoaneurysm of the ascending aorta following cardiac surgery. Chest 93:138-43.

Tavora F, Burke A. 2006. Review of isolated ascending aortitis: differential diagnosis, including syphilitic, Takayasu's and giant cell aortitis. Pathology 38(4):302-308.

Tomey MI, Murthy VL, Beckman JA. 2011. Giant syphilitic aortic aneurysm: a case report and review of the literature. Vasc Med 16(5):360-364.

Watanabe T, Miyazawa K, Tsukamoto M, Shibota Y, Katori R. 1967. Syphilitic aneurysm of the ascending aorta producing pulmonic stenosis by compression. Am J Cardiol 20(4):575-57

S.-M. Yuan. 2018 Syphilitic aortic aneurysm Zeitschrift für Rheumatologie. Volume 77, Issue 8, pp 741-748. 